\title{
Reversible posterior leukoencephalopathy syndrome following combinatorial cisplatin and pemetrexed therapy for lung cancer in a normotensive patient: A case report and literature review
}

\author{
CHANGQING XIE and VOVANTI T. JONES \\ Department of Internal Medicine, Brody School of Medicine, East Carolina University, \\ Vidant Medical Center, Greenville, NC 27834, USA
}

Received December 25, 2014; Accepted September 24, 2015

DOI: $10.3892 / 01.2015 .4059$

\begin{abstract}
Reversible posterior leukoencephalopathy syndrome (RPLS) is a rare neurological syndrome of the brain, causing symptoms such as headaches, seizures, altered mental status and visual disturbances. The condition is predominantly associated with hypertension, eclampsia, renal impairment, cytotoxic drugs, immunosuppressive agents and molecular targeted agents, but the precise underlying mechanism of RPLS is not fully understood. The present study describes the case of a 65 -year-old female patient with stage IIA non-small cell lung cancer who received cisplatin/pemetrexed treatment at the Leo W. Jenkins Cancer Center. Following 3 cycles of this therapy, the patient was referred to the Emergency Department of Vidant Medical Center with an altered mental status, subsequently presenting with epileptic seizures, a fever and a headache. A neurological examination revealed generalized hyperreflexia and paraparesis, with extensor posturing of the bilateral lower extremities. The lumbar puncture and electroencephalography results were normal, but cranial computed tomography (CT) scans revealed attenuation abnormalities in the bilateral parietal region and the left occipital lobe, with suspected metastasis. Cranial T2-weighted magnetic resonance imaging (MRI) indicated bilateral regions of increased signal intensity in the occipital, temporal and periventricular white
\end{abstract}

Correspondence to: Dr Changqing Xie, Department of Internal Medicine, Brody School of Medicine, East Carolina University, Vidant Medical Center, 600 Moye Boulevard, Greenville, NC 27834, USA

E-mail: xiec@ecu.edu

Abbreviations: RPLS, reversible posterior leukoencephalopathy syndrome; NSCLC, non-small cell lung cancer; CT, computed tomography; MRI, magnetic resonance imaging

Key words: reversible posterior leukoencephalopathy syndrome, cisplatin, pemetrexed matter. The patient was treated with anticonvulsants, steroids and antihypertensive drugs, recovered gradually from the symptoms and regained full consciousness. However, the patient reported residual weakness, presenting with an Eastern Cooperative Oncology Group score of 3, reflective of an inability to independently perform daily activities and self-care. A brain MRI performed 10 days later demonstrated that the subcortical edema had partially subsided. The patient was discharged on day 15 post-admission. A follow-up cranial CT examination 1 month later indicated a partial resolution of the abnormalities. The present report reviews similar associated cases, and also discusses the clinical features and mechanisms underlying RPLS. Although it is typically reversible, RPLS is a serious and potentially life-threatening adverse condition if left untreated. Early recognition of this condition is crucial for the prompt control of the patient's blood pressure or withdrawal of cytotoxic drugs in order to reverse this syndrome.

\section{Introduction}

Reversible posterior leukoencephalopathy syndrome (RPLS) was first described by Hinchey et al (1) as an acute illness that causes symptoms such as hypertension, headaches, seizures, altered mental status and visual disturbances, and which is usually reversible following the removal of the causative agents and the control of the patient's blood pressure. RPLS is characterized by white matter edema, particularly involving the bilateral occipital and posterior parietal lobes of the brain (1). Involvement of other areas of the brain, including the frontal lobes, cerebellum, basal ganglia and brain stem, has also been reported.

RPLS is primarily associated with hypertension, eclampsia, renal impairment, cytotoxic drugs, immunosuppressants and molecular targeted agents. The list of common antineoplastic drugs that predispose patients to RPLS is expanding, and includes cisplatin, L-asparaginase, thalidomide, vinflunine, methotrexate, vincristine and cytarabine (2-8). Certain combination regimens have also been associated with RPLS, including a combination treatment of ziv-aflibercept with cisplatin and pemetrexed, the cyclophosphamide, hydroxydaunorubicin, Oncovin and Prednisone 
regimen, intrathecal methotrexate and intravenous ifosfamide, idarubicine and etoposide, bevacizumab/folinic acid, fluorouracil and irinotecan, and capecitabine and cyclophosphamide (9-13).

The mechanisms underlying RPLS have been postulated to be either severe hypertension leading to failed auto-regulation and endothelial injury/vasogenic edema, or vasoconstriction leading to brain ischemia and subsequent vasogenic edema. However, the mechanism by which cytotoxic agents cause RPLS in a normotensive environment is not fully understood, but the disruption of the blood-brain barrier is suspected to be a major contributory factor (14).

The present report describes a normotensive patient who had received cisplatin/pemetrexed for treatment of non-small cell lung cancer (NSCLC) and who subsequently developed RPLS, but was able to recover following treatment. Written informed consent was obtained from the patient's family.

\section{Case report}

The current report describes the case of a 65-year-old female patient that presented to the Leo W. Jenkins Cancer Center (Greenville, NC, USA) in July 2014 with stage IIA NSCLC [tumor-node-metastasis staging score, T1N1M0 (15)] on the left upper lobe, with an enlarged left hilar lymph node. The patient received cisplatin/pemetrexed $\left(75\right.$ and $50 \mathrm{mg} / \mathrm{m}^{2}$, respectively) by intravenous administration, as neoadjuvant chemotherapy, every 3 weeks. However, 3 days after the third cycle of this therapy, the patient was referred to the Emergency Department of Vidant Medical Center (Greenville, NC, USA)presenting with progressive confusion, followed by tonic-clonic seizures, a fever, abdominal pain and a headache. Neurological examination indicated a limited attention span, disorientation, generalized hyperreflexia and paraparesis with extensor posturing of the bilateral lower extremities and reduced dorsiflexion capability. The blood pressure of the patient was $137 / 89 \mathrm{mmHg}$ (normal range, $100-140 / 60-90 \mathrm{mmHg}$ ). Biological investigations at admission revealed a normal white blood cell count and platelet count, borderline hyponatremia (134 mmol/1; normal range, $135-145 \mathrm{mmol} / \mathrm{l}$ ) and mildly elevated ammonia levels (45 mmol/1; normal range, 11-35 mmol/l). Electroencephalography and a lumbar puncture gave normal results, with an opening pressure of $140 \mathrm{~mm} \mathrm{H}_{2} \mathrm{O}$, and no organisms were cultured. Cranial computed tomography (CT) scans revealed attenuation abnormalities on the bilateral parietal region and left occipital lobe, with suspected metastasis. Cranial T2-weighted magnetic resonance imaging (MRI) revealed bilateral areas of increased signal intensity in the occipital, temporal and periventricular white matter (Fig. 1A).

The patient experienced multiple generalized seizures following admission, which were resolved by lorazepam treatment ( $2 \mathrm{mg}$, every $2 \mathrm{~h}$ as required). Anticonvulsants (levetiracetam; 1,500 mg every $12 \mathrm{~h}$ ), dexamethasone (4 mg, every $6 \mathrm{~h}$ ) and antihypertensive agents (amlodipine, $5 \mathrm{mg}$ daily; metoprolol, $25 \mathrm{mg}$ twice daily) were then administered as required in order to treat the remaining symptoms. The patient's mental condition gradually recovered and full consciousness was regained, but the patient reported residual weakness with an ECOG score of 3, reflective of poor ability
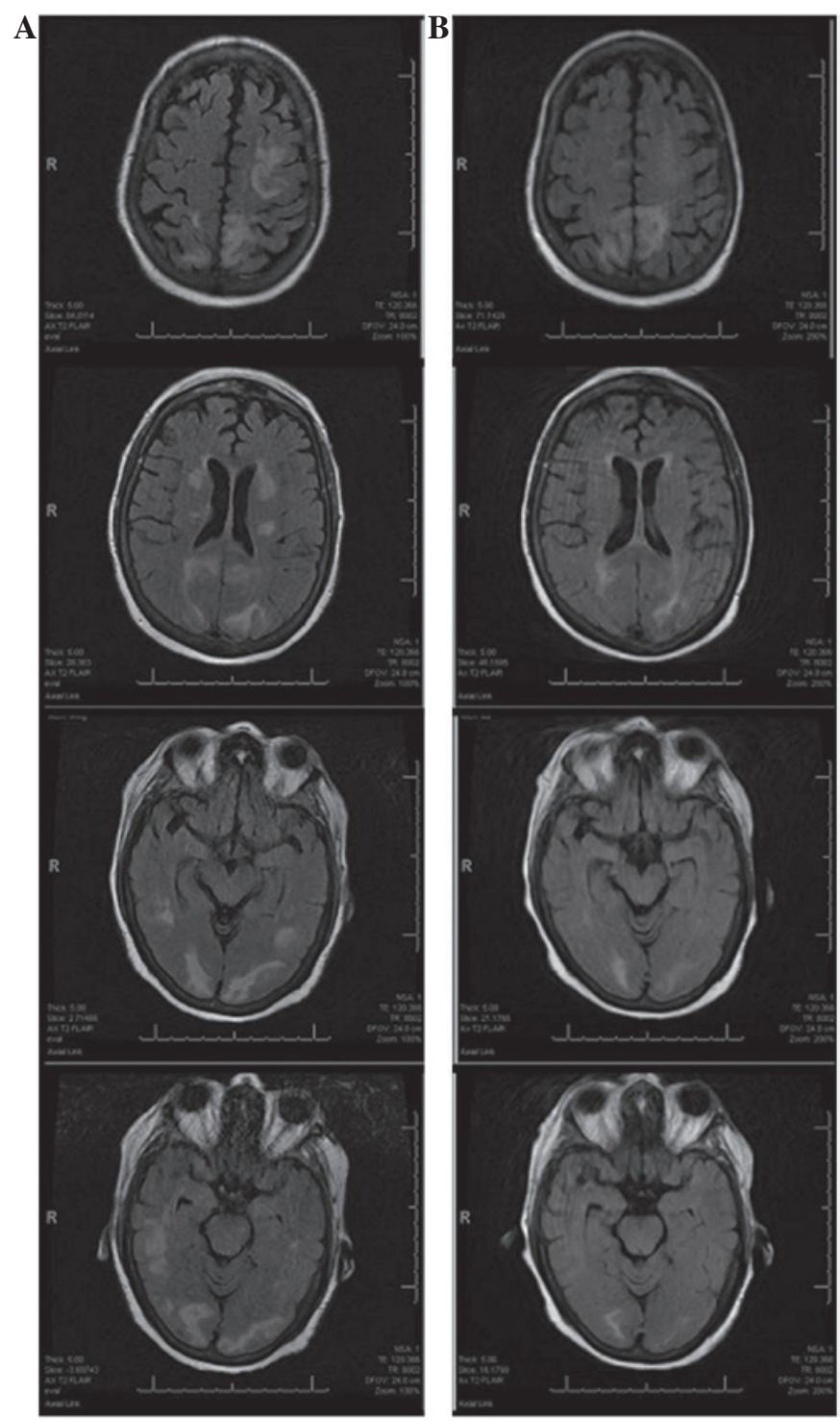

Figure 1. Reversal of diffusion abnormalities in a 65-year-old woman with reversible posterior leukoencephalopathy syndrome associated with cisplatin/pemetrexed treatment. T2-weighted magnetic resonance imaging indicated bilateral regions of increased signal intensity in the occipital, temporal and periventricular white matter (A) upon admission and (B) during the follow-up examination 10 days later, following supportive and symptomatic therapy.

to function with regard to daily activity and self-care. A brain MRI scan performed 10 days later indicated partial alleviation of the subcortical edema (Fig. 1B). The patient was discharged on the day 15 post-admission, and a follow-up cranial CT examination 1 month later demonstrated improved, but not complete, resolution of the abnormalities. The patient succumbed to the disease in September 2014.

\section{Discussion}

The present study was unable to confirm whether cisplatin or pemetrexed was responsible for the onset of RPLS, but there are multiple previous studies of cisplatin-associated RPLS, whether alone or in combinatorial treatments alongside other cytotoxic drugs $(5,16-23)$. However, to the best of 


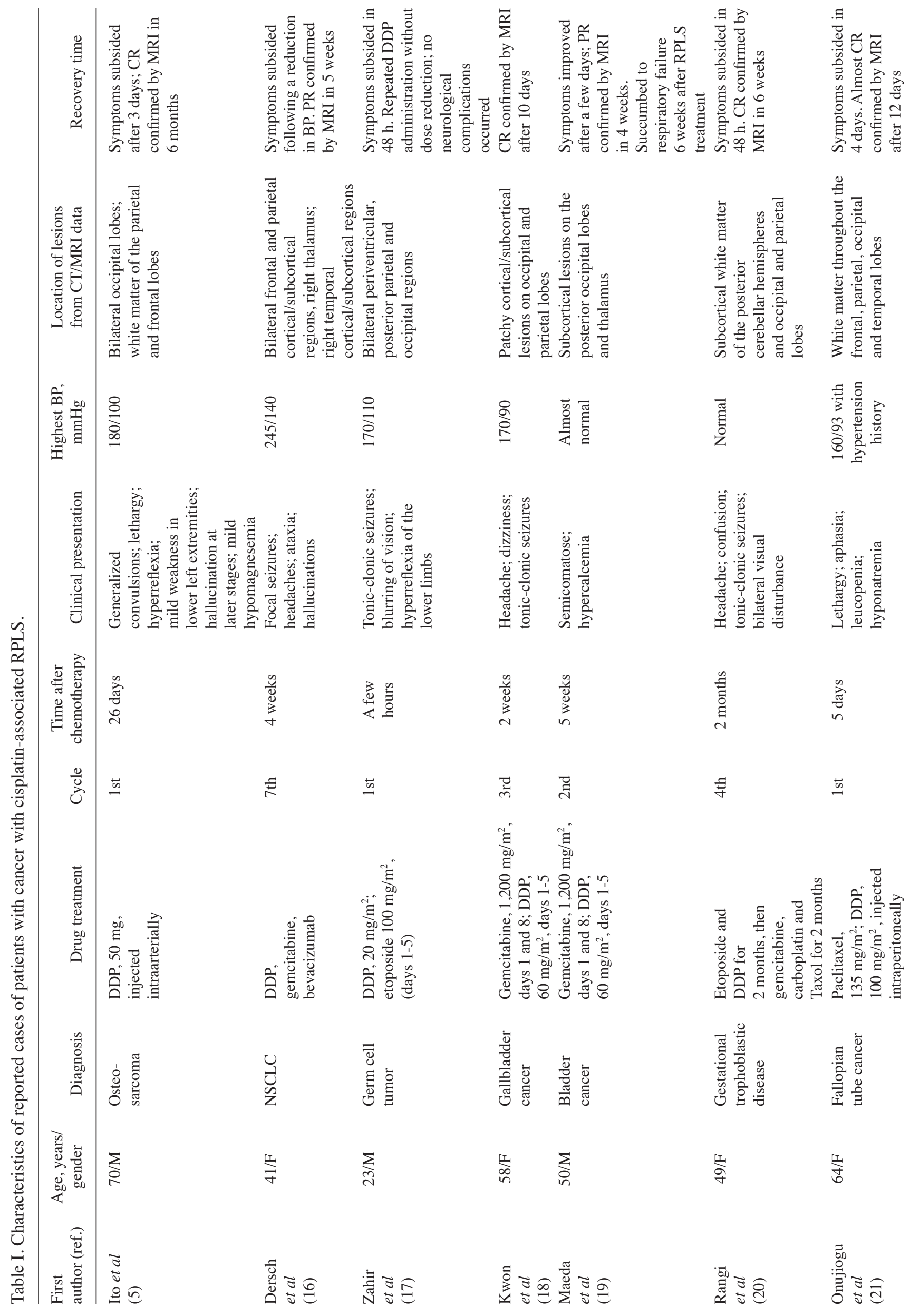




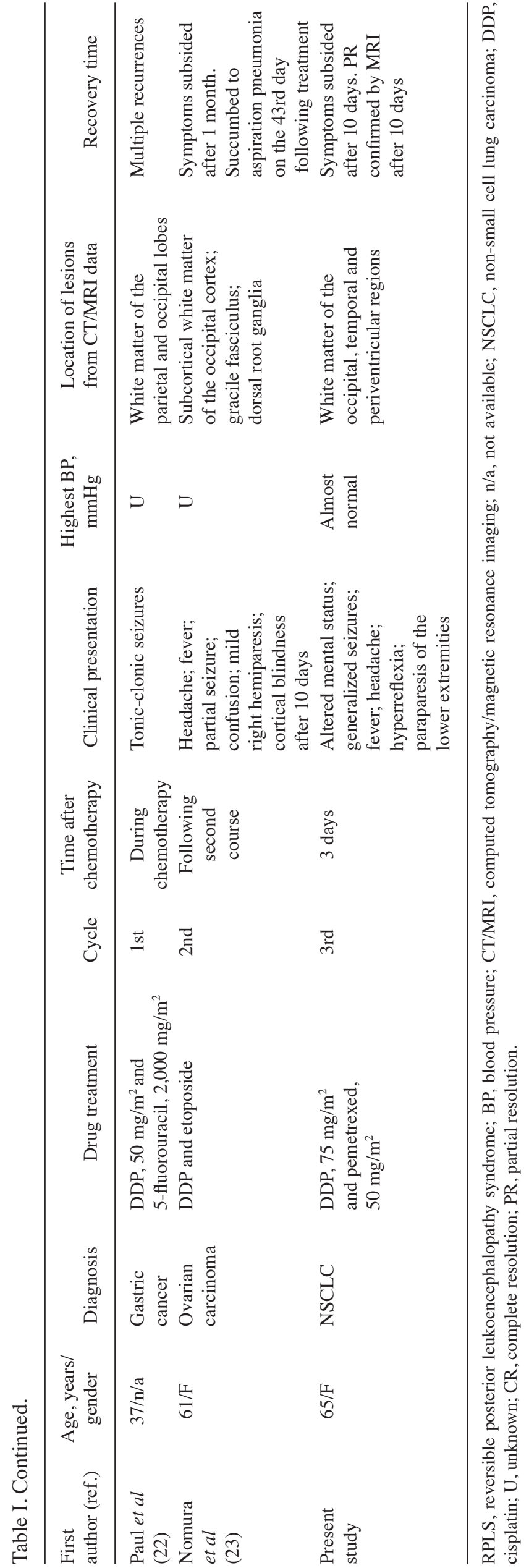

our knowledge, no studies have indicated that pemetrexed alone can induce RPLS; it is therefore conceivable that cisplatin is predominantly responsible for the RPLS reported in the present study.

RPLS is a rare neurological syndrome of the brain that was first defined by Hinchey et al in 1996 (1). The condition is reported in numerous patients with eclampsia, acute hypertensive encephalopathy associated with renal disease and those receiving immunosuppressive therapy or interferon. An association with cisplatin administration in cancer patients has been widely reported in previous studies (Table I) $(5,16-23)$, predominantly occurring within the first 3 cycles of chemotherapy, with the exception of one late-onset case in the 7th cycle (16). In the majority of cases, patients develop hypertension, and additionally present with headaches, seizures, altered mental status and visual disturbances, whilst normotension is only observed in a few cases (5,16-18). Cranial CT/MRI usually indicates cortical/subcortical edema in the bilateral occipital and parietal lobes; uncommon areas in which to observe legions include the thalamus, the cerebellum, the periventricular regions, and the frontal and temporal lobes. Symptoms typically improve rapidly upon gaining control of the patient's blood pressure, upon treatment with anticonvulsants and/or upon withdrawal of cytotoxic drugs. It is notable that this syndrome has serious and potentially life-threatening adverse effects if left untreated.

The precise pathophysiology of RPLS is incompletely understood. As hypertension presents in the majority of patients with RPLS, hypertensive encephalopathy is a probable mechanism of its development. Sudden elevations in systemic blood pressure disrupt the blood-brain barrier, causing the local exchange of fluids. The cerebral white matter is composed of myelinated fiber tracts in an extracellular matrix containing glial cells, arterioles and capillaries, and is susceptible to vasogenic edema (1). The carotid vessels are supplied with a greater number of sympathetic adrenergic innervations than those of the vertebral-basilar system; this inherent deficiency of sympathetic adrenergic innervation may inhibit the vasoconstriction of posterior cerebral vessels, making them prone to RPLS development (24). In normotensive patients with RPLS, including the present case, another possible hypothesis is that damage to the endothelial cells of cerebral vessels by cytotoxic drugs destroys the blood-brain barrier, as proposed in a rat model (14). Numerous RPLS patients have demonstrated metabolic abnormalities, including fever, leukocytosis, hyponatremia, hypocalcemia and hypomagnesemia (2-8), implying that metabolic abnormalities may disturb the integrity of the blood-brain barrier and lead to cerebral edema. In a previous study, a postmortem examination of a patient with cisplatin-associated RPLS indicated severe nerve cell loss, gliosis and spongy changes to the bilateral occipital cortex, including the visual field; furthermore, mild to moderate demyelination in the subcortical white matter of the occipital cortex, gracile fasciculus and dorsal root ganglia was observed. Notably, platinum was detected in the bilateral occipital cortex, spinal cord and cauda equina, suggesting that platinum may contribute to the damaging effects of RPLS (23).

The use of an intravenous cisplatin/pemetrexed regimen can be associated with RPLS; although typically reversible, this syndrome is serious and can be fatal if left untreated. Early recognition is vital in order to control the blood pressure or to withdraw cytotoxic drugs in a timely manner for the 
reversal of RPLS. The precise underlying mechanism of RPLS is not fully understood and is posited to be multifactorial, but it is likely to be associated with the integrity of the blood-brain barrier.

\section{References}

1. Hinchey J, Chaves C, Appignani B, Breen J, Pao L, Wang A, Pessin MS, Lamy C, Mas JL and Caplan LR: A reversible posterior leukoencephalopathy syndrome. N Engl J Med 334: 494-500, 1996.

2. Chow S, Cheung CS, Lee DH, Howson-Jan K and Xenocostas A: Posterior reversible encephalopathy syndrome in a patient with multiple myeloma treated with thalidomide. Leuk Lymphoma 53: 1003-1005, 2012.

3. Helissey C, Chargari C, Lahutte M, Ricard D, Vedrine L, Ceccaldi B and Le Moulec S: First case of posterior reversible encephalopathy syndrome associated with vinflunine. Invest New Drugs 30: 2032-2034, 2012.

4. Hualde Olascoaga J, Molins Castiella T, Souto Hernández S, Becerril Moreno F, Yoldi Petri ME, Sagaseta de Ilurdoz M and Molina Garicano J: Reversible posterior leukoencephalopathy: Report of two cases after vincristine treatment. An Pediatr (Barc) 68: 282-285, 2008 (In Spanish).

5. Ito Y, Arahata Y, Goto Y, Hirayama M, Nagamutsu M, Yasuda T, Yanagi T and Sobue G: Cisplatin neurotoxicity presenting as reversible posterior leukoencephalopathy syndrome. AJNR Am J Neuroradiol 19: 415-417, 1998.

6. Patel A, Ayto R and MacDonald DH: Posterior reversible encephalopathy after intrathecal methotrexate therapy in diffuse large B-cell lymphoma. Br J Haematol 161: 607, 2013.

7. Rathi B, Azad RK, Vasudha N, Hissaria P, Sawlani V and Gupta RK: L-asparaginase-induced reversible posterior leukoencephalopathy syndrome in a child with acute lymphoblastic leukemia. Pediatr Neurosurg 37: 203-205, 2002.

8. Saito B, Nakamaki T, Nakashima H, Usui T, Hattori N, Kawakami K and Tomoyasu S: Reversible posterior leukoencephalopathy syndrome after repeat intermediate-dose cytarabine chemotherapy in a patient with acute myeloid leukemia. Am J Hematol 82: 304-306, 2007.

9. Abali H, Eren OO, Dizdar O, Karada ̌̆ O, Erman M, Yilmaz A Uluç K, Erdem I and Türker A: Posterior leukoencephalopathy after combination chemotherapy in a patient with lymphoma. Leuk Lymphoma 46: 1825-1828, 2005.

10. Allen JA, Adlakha A and Bergethon PR: Reversible posterior leukoencephalopathy syndrome after bevacizumab/FOLFIRI regimen for metastatic colon cancer. Arch Neurol 63: 1475-1478, 2006.

11. Chen H, Modiano MR, Neal JW, Brahmer JR, Rigas JR, Jotte RM, Leighl NB, Riess JW, Kuo CJ, Liu L, et al: A phase II multicentre study of ziv-aflibercept in combination with cisplatin and pemetrexed in patients with previously untreated advanced/metastatic non-squamous non-small cell lung cancer. Br J Cancer 10: 602-608, 2014.
12. Edwards MJ, Walker R, Vinnicombe S, Barlow C, MacCallum P and Foran JM: Reversible posterior leukoencephalopathy syndrome following CHOP chemotherapy for diffuse large B-cell lymphoma. Ann Oncol 12: 1327-1329, 2001.

13. Yasaki S, Tukamoto Y, Yuasa N, Ishikawa T and Yoshii F: Late-onset leukoencephalopathy induced by long-term chemotherapy with capecitabine and cyclophosphamide for liver metastasis from breast cancer. Rinsho Shinkeigaku 52: 251-256, 2012 (In Japanese)

14. Sugimoto S, Yamamoto YL, Nagahiro S and Diksic M: Permeability change and brain tissue damage after intracarotid administration of cisplatin studied by double-tracer autoradiography in rats. J Neurooncol 24: 229-240, 1995.

15. Edge SB, Byrd DR, Compton CC, et al (eds.): Lung. In: AJCC Cancer Staging Manual. 7th edition. Springer, New York, NY, pp253-270, 2010.

16. Dersch R, Stich O, Goller K, Meckel S, Dechent F, Doostkam S, Weiller C and Bardutzky J: Atypical posterior reversible encephalopathy syndrome associated with chemotherapy with Bevacizumab, Gemcitabine and Cisplatin. J Neurol 260: 1406-1407, 2013.

17. Zahir MN, Masood N and Shabbir-Moosajee M: Cisplatin-induced posterior reversible encephalopathy syndrome and successful re-treatment in a patient with non-seminomatous germ cell tumor: A case report. J Med Case Rep 6: 409, 2012.

18. Kwon EJ, Kim SW, Kim KK, Seo HS and Kim do Y: A case of gemcitabine and cisplatin associated posterior reversible encephalopathy syndrome. Cancer Res Treat 41: 53-55, 2009.

19. Maeda T, Kikuchi E, Matsumoto K, Yazawa S, Hagiuda J, Miyajima A, Nakagawa K, Fujiwara H, Hoshino $\mathrm{H}$ and Oya M: Gemcitabine and cisplatin chemotherapy induced reversible posterior leukoencephalopathy syndrome in a bladder cancer patient. Int J Clin Oncol 15: 508-511, 2010.

20. Rangi PS, Partridge WJ, Newlands ES and Waldman AD: Posterior reversible encephalopathy syndrome: A possible late interaction between cytotoxic agents and general anaesthesia. Neuroradiology 47: 586-590, 2005.

21. Onujiogu N, Lengyel E and Yamada SD: Reversible posterior leukoencephalopathy syndrome following intravenous paclitaxel and intraperitoneal cisplatin chemotherapy for fallopian tube cancer. Gynecol Oncol 111: 537-539, 2008.

22. Paul F, Aktas O, Dieste FJ, Kreitsch P, Vogel HP and Zipp F: Relapsing reversible posterior leukoencephalopathy after chemotherapy with cisplatin and 5-fluorouracil. Nervenarzt 77: 706-710, 2006 (In German).

23. Nomura K, Ohno R, Hamaguchi K, Hata T, Hatanaka H and Matsuyama H: Clinicopathological report of cisplatin encephalopathy. Rinsho Shinkeigaku 35: 64-69, 1995 (In Japanese).

24. Perloff D: Hypertension and pregnancy-related hypertension. Cardiol Clin 16: 79-101, 1998. 